\title{
Study on the extraction and antibacterial activity of Monascin
}

\author{
Xiaojuan $\mathrm{Gao}^{1}$, Xiaoshi $\mathrm{Lu}^{1}$, Zifeng Wang ${ }^{1}$, Guangpeng $\mathrm{Liu}^{2}$ and Xinjun $\mathrm{Li}^{1, * *}$ \\ ${ }^{1}$ Qilu Institute of Technology, Jinan, Shan Dong, 250200, China \\ ${ }^{2}$ Jinan Fruit Research Institute, All-China Federation of Supply and Marketing Cooperatives, Jinan, Shan Dong, 250200, China
}

\begin{abstract}
Taking monascin as the research object, monascin was extracted from red kojic rice by ethanol extraction and extracted with $60 \%, 70 \%$ and $80 \%$ ethanol respectively. Finally, it was concluded that when the concentration of ethanol was $70 \%$, the extraction rate of monascin was the highest, reached $75.68 \%$. The bacteriostatic experiments of monascin extract and monascin fermentation showed that it had strong inhibitory effect on Staphylococcus aureus and Bacillus subtilis, weak inhibitory ability on Escherichia coli and Aspergillus niger, and no obvious inhibitory effect on the growth of Saccharomyces cerevisiae.
\end{abstract}

\section{Introduction}

Monascus purpureus belongs to fungal kingdom, Ascomycota, Ascomycetes, Eurotiales, Monascaceae, Monascus in taxonomy. Monascus purpureus is suitable for growing on wort Agar medium. The colonies are white at the initial stage, turn red after ripening, and produce red pigment ${ }^{[1]}$. The red pigment is a secondary metabolite produced by Monascus and it is a natural pigment ${ }^{[2]}$. Monascus pigment has the advantages of good thermal stability, strong protein coloring power, soft tone and stable to acid and alkali, and Monascus pigment has no toxicity, no mutagenicity and high safety ${ }^{[3-4]}$. In East Asia, the red pigment produced by Monascus is also widely used in food dyeing ${ }^{[5]}$.In the fermentation broth of Monascus, there are components with antibacterial activity, which are collectively referred to as Monascin. Chinese people have a long tradition of using Monascus to preserve food, which is actually beneficial to the bacteriostatic performance of Monascus. Japanese people has already begun to use Monascus pigment for the preservation of fish and meat, and has obtained a lot of patents ${ }^{[6]}$. Monascin has a strong bactericidal effect on some bacteria which provides a new idea for the treatment of some diseases and food preservation ${ }^{[7]}$. The best ethanol extraction concentration of erythromycin was obtained by measuring the extraction rate of monascin. The bacteriostatic effect of monascin extract and Monascus purpureus fermentation broth was also studied in order to provide data support for the bacteriostatic application of Monascus purpureus.

\section{Materials and methods}

\subsection{Materials and reagents}

Red kojic rice: purchased in the market; Monascus purpureus: isolated from Red kojic rice; sensitive strain: laboratory preserved strain; sucrose (A.R): China National Pharmaceutical Group Shanghai Reagent Co.Ltd.; peptone (B.R), Beef extract (B.R), Yeast extract (B.R): Beijing Oboxing Biotechnology Co.Ltd.; glucose (A.R): Tianjin Damao Chemical Reagent Factory. Sodium Chloride (A.R): Tianjin Damao Chemical Reagent Factory; Agar Powder (A.R): Tianjin Beichen Fangzheng Reagent Factory; Nutrition Agar (A.R): Hangzhou Best Biotechnology Co.Ltd.

\subsection{Instruments and equipment}

SW-CJ-2F ultra-clean workbench: Suzhou Purification Equipment Co.Ltd.; 721 visible spectrophotometer: Shanghai Hengping Scientific Instrument Co.Ltd.; HHW2 electric thermostatic water bath pot: Beijing Yongming Medical Instrument Factory; SHZ-82 digital display constant temperature air bath oscillator: Changzhou Guohua Electric Appliance Co.Ltd.; YXQLS-S II automatic vertical electrothermal pressure steam sterilizer: Medical Equipment Factory of Shanghai Boxun Industrial Co.Ltd

\section{Experimental methods}

\subsection{Isolation and purification of Monascus purpureus}

$5.0 \mathrm{~g}$ red koji rice ${ }^{[8]}$ mixed with $45 \mathrm{~mL}$ sterile water and kept in a constant temperature water bath at $60^{\circ} \mathrm{C}$ for 30 minutes to remove heat-labile bacteria and yeasts from the samples. The bacterial solution was evenly coated on aseptic wort Agar medium and cultured at $28^{\circ} \mathrm{C}$ for 5 days. The single colony of mold producing red pigment was selected and observed under microscope. After it was confirmed as pure Monascus purpureus, low-temperature

\footnotetext{
"Corresponding author: 328449142@qq.com
} 
preservation slant.

\subsection{Determination of color value of Monascin}

Put $0.5 \mathrm{~g}$ red koji rice mixed with $1 \mathrm{~mL} 70 \%$ ethanol for grinding. Pour the grinding fluid into the plug test tube, wash the mortar and pestle twice with $70 \%$ ethanol $6 \mathrm{~mL}$, and pour the washing liquid into the test tube. The test tube was placed in a constant temperature water bath at $60^{\circ} \mathrm{C}$ for extraction for 30 minutes and vibrated every 5 minutes. Then cool and filter, wash the residue twice with $70 \%$ ethanol solution, combine all filtrates, and fix the volume to $10 \mathrm{~mL}$ with $70 \%$ ethanol. The absorbance of A505 was determined at $505 \mathrm{~nm}$ with $70 \%$ ethanol as reference. According to the formula: monascus color value (calculated by $1 \mathrm{~g}$ sample $)=\mathrm{A} 505 \times$ sample volume $(\mathrm{mL}) /$ sample mass $(\mathrm{g}) \times 100 \%$.

\subsection{Extraction of Monascin}

Put $6.0 \mathrm{~g}$ red koji rice mixed with $60 \%$ ethanol for grinding. Pour the grinding fluid into the flask of rotary evaporator, washed the mortar with ethanol solution for 3 times, and pour the washing solution into the flask for rotary distillation extraction. Extract the solution from bright red to dark red, filter the extract, and continue to add $60 \%$ ethanol solution for extraction. Repeat the above operation for 4 times and merge the filtered extract. Then $70 \%$ and $80 \%$ ethanol solution were used for the above extraction operation respectively, and three different monascin extracts were obtained. The absorbance of three kinds of monascin solution was determined under $505 \mathrm{~nm}$ with $60 \%$, $70 \%$ and $80 \%$ ethanol solution as reference respectively. Finally, according to the color value of red koji rice samples, the extraction rate of monascin was calculated by spinning three kinds of ethanol concentration, and the optimal ethanol extraction concentration was obtained by analysis and comparison.

\subsection{Fermentation experiment of Monascus purpureus $^{[9]}$}

The strains isolated and purified from Monascus purpureus were inoculated into the seed fermentation medium and shaken at $180 \mathrm{r} / \mathrm{min}$ at $28^{\circ} \mathrm{C}$ for 2 days and at $37^{\circ} \mathrm{C}$ for 2 days. $10 \%$ inoculum was inoculated into Monascus purpureus fermentation medium, $180 \mathrm{r} / \mathrm{min}$, and the mycelium growth was observed after shaking culture at $28^{\circ} \mathrm{C}$ for 2 days and shaking culture at $37^{\circ} \mathrm{C}$ for 4 days.

\subsection{Determination of bacteriostatic activity of monascin $^{[10]}$}

(1) Cylinder plate method: $200 \mu \mathrm{L}$ Staphylococcus aureus suspension was spread evenly in nutrient agar medium. Five sterile Oxford cups were placed in the middle of the medium and four points uniformly distributed around the medium with aseptic tweezers. $200 \mu \mathrm{L}$ sterile water was put into the Oxford cup in the middle as blank control; $200 \mu \mathrm{L}$ monascin was put into four Oxford cups around. After incubated at $37^{\circ} \mathrm{C}$ for 24 hours, the bacteriostatic zone was observed and the diameter of the bacteriostatic zone was measured with a scale.

(2) Filter paper method: $200 \mu \mathrm{L}$ Staphylococcus aureus suspension was spread evenly in nutrient agar medium. The round filter paper soaked in aseptic water for 30s with aseptic tweezers was placed in the middle of the culture medium as a blank control, and the round filter paper soaked in monascin for 30s was evenly distributed around the four points. The culture and measurement methods are the same as the cup-plate method.

(3) When the tested bacteria are Escherichia coli and Bacillus subtilis, the method is the same as above. When the tested bacteria were Saccharomyces cerevisiae and Aspergillus niger, they were cultured in modified martin medium at $28^{\circ} \mathrm{C}$ for $48 \mathrm{~h}$.

Repeat the above experimental steps, replace the bacteriostatic solution with Monascus purpureus fermentation broth, and compare the bacteriostatic ability of Monascus purpureus fermentation broth and monascin.

\section{Results and discussion}

\subsection{Extraction of monascin}

(1) Determination of primary color value of original monascin

Table 1. Determination of primary color value of original monascin.

\begin{tabular}{lllll}
\hline Sample & $70 \%$ ethanol & No. 1 & No. 2 & No. 3 \\
\hline A $_{505}$ & 0 & 1.941 & 1.884 & 1.790 \\
\hline \multicolumn{7}{l}{ The color value of original monascin } & $0.37 \%$ \\
\hline
\end{tabular}

(2) determination of color value of monascin extracted by ethanol.

With $60 \%, 70 \%$ and $80 \%$ concentration of ethanol as the standard sample, the extraction $3.0 \mathrm{~g}$ sample was diluted to $25 \%$ of the absorbance value. The color values of three kinds of monascin are calculated according to the above color value formula, and the three color values obtained are divided by the original color value of monascin, and the extraction rates of three kinds of monascin are calculated, as shown in Table 2:

Table 2.absorbance value and extraction rate of monascin extracted with three concentrations of ethanol.

\begin{tabular}{cccc}
\hline Sample & A505 & color value & extraction \\
\hline $60 \%$ ethanol & 0 & & \\
No. 1 & 1.890 & $0.25 \%$ & $67.57 \%$ \\
No. 2 & 1.960 & & \\
No. 3 & 1.841 & & \\
$70 \%$ ethanol & 0 & & \\
No. 1 & 2.062 & $0.28 \%$ & $75.68 \%$ \\
No. 2 & 2.066 & & \\
No. 3 & 2.060 & & \\
$80 \%$ ethanol & 0 & & \\
No. 1 & 1.951 & $0.26 \%$ & $70.27 \%$ \\
No. 2 & 1.951 & & \\
No. 3 & 1.948 & & \\
\hline
\end{tabular}

It can be calculated from the above data that monascin 
can be obtained by ethanol rotary steam extraction. the relationship between the concentration of ethanol and the extraction rate of monascin was a quadratic function parabola, and the parabola peak was at the concentration of about $70 \%$ ethanol. The extraction rate of monascin was the highest and the effect is the best.

\subsection{Monascus purpureus fermentation}

Monascus purpureus was inoculated into the seed liquid medium for culture, and the white mycelium ball could be clearly observed after 4 days, as shown in Fig. 1. The seed liquid was inoculated into Monascus purpureus fermentation medium and the formula of double rice flour was used to culture the white mycelium ball which was larger and more round than that in the seed culture medium. Part of the bacterial liquid is attached to the inner wall of the flask and has begun to produce red pigment, as shown in Fig. 2.

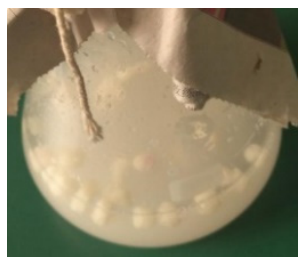

Fig. 1. seed mycelium ball of Monascus purpureus.
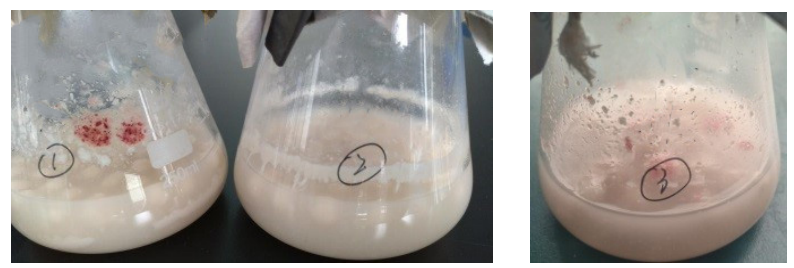

Fig. 2. mycelium ball fermented by Monascus purpureus.

\subsection{Inhibition on Staphylococcus aureus}

In Staphylococcus aureus, the inhibitory effect of monascin extract is very obvious. The average diameter of bacteriostatic zone in cylinder plate method is $1.90 \mathrm{~cm}$, as shown in the left of Fig. 3, and the average diameter of bacteriostatic zone in filter paper method is $1.50 \mathrm{~cm}$.

Monascus purpureus fermentation broth has obvious inhibitory effect on Staphylococcus aureus. The average diameter of the bacteriostatic zone of the left cylinder plate method can reach $1.50 \mathrm{~cm}$, as shown in the right picture of Fig.3, and the average diameter of the bacteriostatic zone of the filter paper method is $1.10 \mathrm{~cm}$.
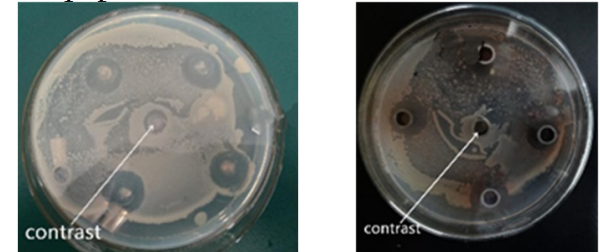

Fig. 3. Bacteriostasis to Staphylococcus aureus.

\subsection{Inhibition on Bacillus subtilis}

For Bacillus subtilis, the inhibitory effect of monascin extract was very obvious. The average diameter of bacteriostatic zone in cylinder plate method is $2.00 \mathrm{~cm}$, as shown in the left of Fig. 4, and the average diameter of bacteriostatic zone in filter paper method is $1.60 \mathrm{~cm}$.

The fermentation broth of Monascus purpureus had obvious inhibitory effect on Bacillus subtilis. The average diameter of bacteriostatic zone in cylinder plate method is $1.50 \mathrm{~cm}$, as shown in the right of Fig. 4 , and the average diameter of bacteriostatic zone in filter paper method is $1.00 \mathrm{~cm}$.
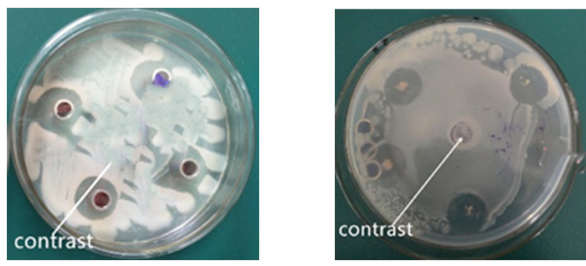

Fig. 4. Bacteriostasis to Bacillus subtilis.
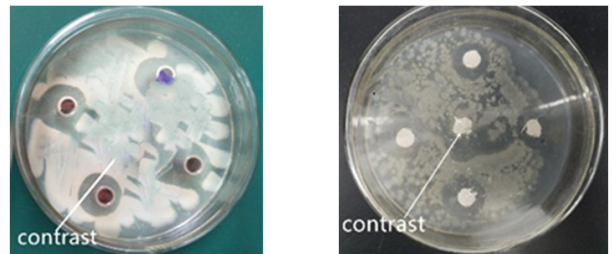

Fig. 5. Bacteriostatic zone of monascin on Bacillus subtilis (left: cylinder plate method; right: filter paper method).

\subsection{Inhibition on Escherichia coli}

For Escherichia coli, the inhibitory effect of monascin extract was not obvious. The average diameter of bacteriostatic zone in cylinder plate method is $1.30 \mathrm{~cm}$, as shown in the left picture of Figure 6, and the average diameter of bacteriostatic zone in filter paper method is $0.90 \mathrm{~cm}$.

The inhibitory effect of Monascus purpureus fermentation broth on Escherichia coli was not obvious. The average diameter of bacteriostatic zone in cylinder plate method is $0.95 \mathrm{~cm}$, as shown in the right picture of Figure 6, and the average diameter of bacteriostatic zone in filter paper method is $0.70 \mathrm{~cm}$.
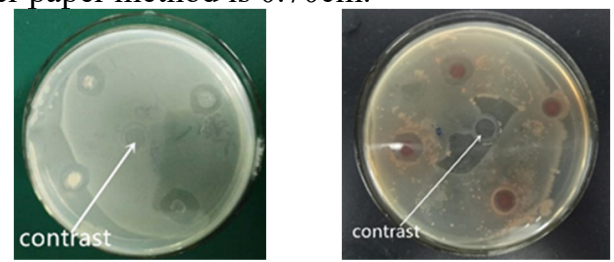

Fig. 6. Bacteriostasis to Escherichia coli.

\subsection{Inhibition on Aspergillus niger}

For Aspergillus niger, monascin had no obvious inhibitory effect and no bacteriostatic zone appeared, as shown on the left side of Figure 7. Under the same culture conditions and time, the number of spores produced by Aspergillus niger by cylinder plate method was significantly less than that of Aspergillus niger by filter paper method.

Monascus purpureus fermentation broth also had no obvious inhibitory effect on Aspergillus niger, and no bacteriostatic zone appeared, as shown in the right side of Fig. 7. Under the same culture conditions and time, Aspergillus niger produced almost no spores, while filter 
paper method produced a small amount of black spores.
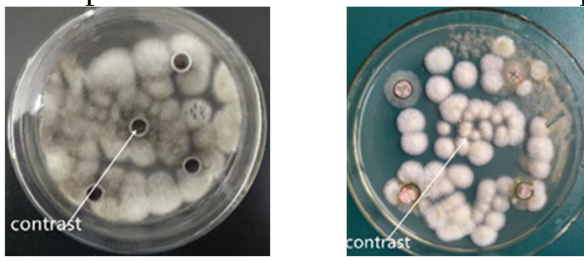

Fig. 7. Bacteriostasis to Aspergillus niger.

\subsection{Inhibition on Saccharomyces cerevisiae}

For Saccharomyces cerevisiae, monascin and Monascus purpureus fermentation broth had no obvious inhibitory effect on its growth, and no bacteriostatic circle appeared, as shown in Fig. 8.
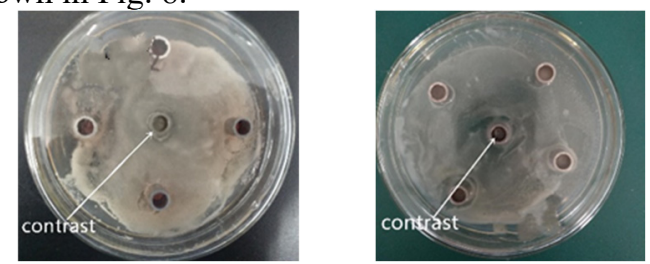

Fig. 8. Bacteriostasis to Saccharomyces cerevisiae.

Meanwhile, it was found that Monascus purpureus fermentation broth only showed some signs of growth in bacterial medium at the same time, but it grew well on fungal medium in Oxford cup and filter paper with fermentation broth. It shows the fluffy characteristics of mold, and even some obvious red pigments.

\section{Results and discussion}

In summary, the bacteriostatic ability of Monascus purpureus fermentation broth to bacteria is worse than that of monascin, the formation rate of bacteriostatic zone is lower, and the diameter of bacteriostatic zone is smaller. It is speculated that there are the following reasons:

(1) Monascin may contain a small amount of ethanol residue, which enhances the bacteriostatic effect;

(2) The fermentation broth of Monascus purpureus is inoculated in nutrient agar medium, which lacks some nutrients and grows poorly. It is not enough to produce enough monascin to inhibit bacteria.

(3) At the same time, when the bacteria had completed the growth around the Oxford cup or filter paper, Monascus purpureus grew slowly due to the influence of the culture medium, and when its growth reached the death of the surrounding colonies caused by sufficient metabolism of monascin, no bacteriostatic results were observed.

(4) Monascus purpureus may produce some metabolites during growth, which have an effect on the bacteriostatic ability of monascin.

\section{References}

1. Ghosh Sandip, et al. Genome shuffling improves pigment and other bioactive compound production in Monascus purpureus. Applied Microbiology and Biotechnology, 104(24):10451-10463 (2020)

2. Zhiqiang Li, Ying Liu, et al. Screening and identification of Monascus strains with high-yield monacolin $\mathrm{K}$ and undetectable citrinin by integration of HPLC analysis and pksCT and ctnA genes amplification. Journal of applied microbiology, 129(5):1410-1418 (2020)

3. Bernhard Thalhamer, et al. Adulteration of beetroot red and paprika extract based food colorant with Monascus red pigments and their detection by HPLC-QTof MS analyses. Food Control, 105:58-63 (2019)

4. Xuelian Yang, Longbei Xiang, et al. Effect of nonionic surfactant Brij 35 on morphology, cloud point, and pigment stability in Monascus extractive fermentation. Journal of the Science of Food and Agriculture, 100(12):4521-4530 (2020)

5. Yajun Jiang, Xucong Lv, et al. Microbial dynamics and flavor formation during the traditional brewing of Monascus vinegar. Food Research International, 125 (2019)

6. Xuefeng Zeng, Zhongyue Tang, et al. Effect of red koji as a Starter Culture in "Wanergao": A Traditional Fermented Food in China. Food science \& nutrition, 8(10):5580-5590 (2020)

7. Jia Song, Jiaojiao Zhang, et al. Monascus vinegarmediated alternation of gut microbiota and its correlation with lipid metabolism and inflammation in hyperlipidemic rats.Journal of Functional Foods, 74 (2020)

8. Rabab Hachem, Gaëtan Assemat, Stéphane Balayssac, et al. Comparative Chemical Profiling and Monacolins Quantification in Red Yeast Rice Dietary Supplements by 1H-NMR and UHPLCDAD-MS. Molecules (Basel, Switzerland), 25(2) (2020)

9. Chuannan Long, $\mathrm{Xu}$ Zeng, et al. High-level production of Monascus pigments in Monascus ruber CICC41233 through ATP-citrate lyase overexpression. Biochemical Engineering Journal, 146:160-169 (2019)

10. Victoria Drauch, Claudia Ibesich, Claus Vogl, et al. In-vitro testing of bacteriostatic and bactericidal efficacy of commercial disinfectants against Salmonella Infantis reveals substantial differences between products and bacterial strains. International Journal of Food Microbiology, 328 (2020) 